\title{
Redes de Cooperação como Estratégia para Internacionalização de Pe- quenas e Médias Empresas: $O$ caso da rede Petrogas
}

\author{
Cooperation Networks as Strategy for Internationalization of Small and Medium \\ Enterprises: The case Petrogas network
}

\begin{abstract}
Resumo
As pequenas e médias empresas - PMEs apresentam limitações técnicas e operacionais que geralmente as impedem de ingressar no mercado internacional. Todavia, estas limitações podem ser neutralizadas por meio das redes de cooperação, partindo do pressuposto que a atuação conjunta entre organizações permite obter os recursos necessários para a internacionalização. Diante deste contexto, o presente artigo se propôs a analisar como a Rede de Cooperação da Cadeia Produtiva de Petróleo e Gás de Sergipe - Rede Petrogas contribui para a internacionalização das pequenas e médias empresas - PMEs associadas à referida rede. Após realizar um estudo qualitativo, por meio do método de pesquisa Estudo de Caso, utilizando o modelo analítico adaptado de Brito (1993), evidenciou-se que a rede Petrogas fornece acesso a diversos benefícios estratégicos e operacionais que possibilitam as PMEs, vinculadas à rede, reunir os recursos necessários para atuar no mercado externo, os quais, em caso de atuação isolada, provavelmente não seriam alcançados.
\end{abstract}

Palavras-chave: Rede de cooperação. Internacionalização. Pequenas e médias empresas.

\begin{abstract}
Small and medium enterprises - SMEs have technical and operational limitations that often prevent them from entering the international market. However, these limitations can be neutralized by means of cooperation networks, assuming that the joint action between organizations to deliver the necessary resources for internationalization. Given this context, the present study aimed to analyze how the Cooperation Network of Supply Chain Oil \& Gas of Sergipe - Petrogas Network contributes to the internationalization of small and medium enterprises - SMEs associated with that network. After conducting a qualitative study using the method of case study research using the analytical model adapted de Brito (1993), it was evident that Petrogas network provides access to various strategic and operational benefits that enable SMBs, linked to the network, gather the resources needed to operate in the foreign market, which, if acting alone, would probably not be achieved.
\end{abstract}

Keywords: Cooperation network; Internationalization; Small and medium enterprises.

\footnotetext{
Jefferson Reis Guimarães Andrade ${ }^{1}$, Maria Elena Leon Olave $^{2}$ e Wellington Antonio Santos Silva ${ }^{3}$

${ }^{1}$ Mestre em Administração; Universidade Tiradentes; Rua Modernista, noํ100, Codomínio Parque dos Artista, Bloco Alvaro

Santos, Ap. 404; Aracaju, Brasil - jefferson.comex@hotmail.com

${ }^{2}$ Doutora em Engenharia de Produção; Universidade Federal de Sergipe; Sergipe, Brasil

${ }^{3}$ Especialista em Marketing; Universidade Tiradentes; Aracaju, Brasil
} 


\section{Introdução}

$\mathrm{N}$ as últimas décadas as organizações estão vivenciando rápidos progressos tecnológicos, acompanhados da ampliação da concorrência a nível global e de disputas pelo mercado doméstico com firmas multinacionais (PIRES; MACHADO NETO, 2012; VERDU, 2011).

Frente a este cenário, as empresas estão sendo impulsionadas a buscar alternativas que lhes proporcionem, dentre outros benefícios, melhoria na configuração do seu produto, acesso a modernas tecnologias, aperfeiçoamento dos processos de gestão, redução da dependência do mercado interno, e a conquista de novos mercados (JUCHNIEVSKI1; SOARES, 2013)

De acordo com Caldas et. al. (2010) e Zahra, Ucbasaran e Newey (2009) a internacionalização é a estratégia mais adequada para atingir os objetivos descritos acima, haja vista que o ingresso em mercados externos, além de ampliar o campo de atuação da empresa, impulsiona a organização a investir no aprimoramento de seus recursos e habilidades para manter-se atuando no mercado internacional, o que consequentemente impacta na ampliação do seu potencial competitivo.

No entanto, as empresas de pequeno e médio porte, em virtude de restrições financeiras, tecnológicas e operacionais, geralmente não conseguem ingressar no mercado internacional (HOLLENSTEIN, 2005). Segundo o Ministério do Desenvolvimento, Indústria e Comércio Exterior - MDIC (2012) a participação deste grupo de empresas é de apenas aproximadamente 5\% para as exportações brasileiras, apesar de representarem 99\% das empresas nacionais.

No segmento de petróleo e gás brasileiro, o qual possui cadeia produtiva internacionalizada, observase que o não envolvimento das pequenas empresas em operações internacionais, as impede de firmar parceiras e fornecer produtos e serviços para grandes corporações multinacionais petrolíferas, bem como competir com concorrentes internacionais no mercado doméstico, que vem crescendo de forma exponencial, de $3 \%$ para $13 \%$ do Produto Interno Bruto - PIB do Brasil, no período de 2000 a 2014 (CORTEZIA; SOUZA, 2011; MACCHIA et. al , 2014).

Para superar os fatores limitadores da internacionalização das pequenas empresas, diversos autores ressaltam que os empreendimentos de menor porte podem desenvolver ações conjuntas entre si, a fim de integrar competências e habilidades de diversos parceiros e obter os elementos viabilizares da internacionalização, os quais, em caso de atuação isolada, provavelmente, seriam inacessíveis a estas organizações (ALVARENGA; BALESTRIN, 2009; BOEHE; TONI, 2006; CHETTY; ANGDAL, 2007; CHETTY; CAMPBELL-HUNT, 2004; DALMORO, 2009; FENSTERSEIFER, 2000; JOHNSEN; JOHNSEN, 1999; VERDU, 2011).

Partindo desta acepção, observa-se que no Estado de Sergipe foi criada Rede de Cooperação da Cadeia Produtiva de Petróleo e Gás em Sergipe - Rede PetrogasSE, no ano de 2003, envolvendo a associação de firmas de micro, pequeno e médio porte de varias atividades econômicas, com o propósito de promover a inserção destas empresas na cadeia de petróleo e gás nacional e internacional (MARTINS; SANTANA, 2014).

Diante do exposto, o presente estudo apresenta o objetivo geral de analisar como a rede Petrogas-SE contribui para a internacionalização de pequenas e médias empresas - PMEs; e especificamente busca descrever o perfil da rede de cooperação e das PMEs vinculadas à referida rede que estão internacionalizadas; e avaliar como a cooperação interorganizacional subsidiou a internacionalização destas organizações.

É importante destacar que os objetivos ora propostos visam preencher lacunas literárias de um tema de pesquisa considerado pouco explorado, principalmente pela literatura nacional (DALMORO E WITTMANN, 2011).

Em seguida, apresenta-se o referencial teórico.

\section{Referencial Teórico}

O referencial teórico deste artigo encontra-se dividido em duas seções, contendo a revisão da literatura nacional e internacional sobre redes de cooperação e a sua influencia na internacionalização de pequenas e médias empresas.

2.1 Redes de Cooperação Interorganizacional

O reconhecimento de que as organizações não contemplam sozinhas todos os recursos e competências necessárias para manter-se competitiva e garantir a sua sobrevivência de forma independente é um dos fatores que reforçam a necessidade de desenvolver ações articuladas em redes (REIS et. al, 2008).

No campo organizacional, o conceito de "redes" possui vários enfoques, contemplando a uma grande variedade de formas de relações entre firmas, como clusters, alianças estratégicas, relações de terceirização, subcontratação, distritos industriais, consórcios, franquias, redes de cooperação, entre outras (BALESTRIN; VARGAS, 2004; CÂNDIDO 2002).

As redes de cooperação interorganizacional podem ser definidas como estruturas formadas por empresas interdependentes, que em virtude de limitações de ordem dimensional, financeira e estrutural, não conseguem assegurar isoladamente as devidas condições de sobrevivência e desenvolvimento e, em virtude disto, passam a atuar de forma conjunta na busca de resultados sinérgicos, baseados na complementaridade, compartilhamento, colaboração e conectividade de recursos e capacidades (VERSCHOORE; BALESTRIN, 2008; VINHAS; BECKER, 2006).

Os autores Balestrin, Verschoore e Reyes Junior (2010) e Castro, Bulgacov e Hoffmann (2011) complementam o conceito acima destacando que as redes cooperativas 
são acordos feitos entre atores econômicos (empresas) e que podem envolver atores não econômicos (apoio governamental) que se relacionam com o objetivo de sustentar uma vantagem competitiva, mas sem perder a sua autonomia e identidade.

Nesta égide, Amato Neto (2000) ressalta que a cooperação pode proporcionar o compartilhamento de recursos produtivo; divisão de custos com pesquisas e desenvolvimento; partilha de riscos com a exploração de novas oportunidades e ingresso em mercados internacionais.

A próxima seção apresenta de forma detalhada como as redes de cooperação contribuem para o processo de internacionalização.

\subsection{Redes de cooperação e Internacionalização de Pequenas e Médias Empresas}

De acordo com Floriani e Fleury (2012), a partir do início da década de 1990, o Brasil intensificou a sua inserção na economia internacional, por meio da abertura progressiva de seu comércio exterior e da busca do mercado externo como uma estratégia de crescimento, executada principalmente pelas grandes empresas.

Hoje, as grandes corporações ainda são as responsáveis pelo maior parte do valor faturado no país com transações internacionais. Dados do MDIC (2012) revelam que no ano de 2012, 95\% do valor das exportações brasileiras foi originado por estas companhias.

Oliveira e Martinelli (2005, p. 56) justificam a baixa participação dos pequenos e médios empreendimentos no mercado internacional, argumentando que para estas empresas o processo de internacionalização é mais complexo em comparação com as grandes organizações, devido às dificuldades que lhe são peculiares, tais como: fabricação de produtos com um nível de qualidade aceitável nos mercados externos, falta de tempo para gerenciar as atividades internacionais; falta de informação adequada para se desenvolver no mercado externo; barreiras burocráticas; dificuldade em encontrar intermediários no exterior (agentes de exportação, representantes, empresa comercial), dentre outras.

No entanto, diversos autores afirmam que as dificuldades acima citadas podem ser superadas por meio de estratégias cooperativas entre firmas, fundamentando-se no pressuposto de que a cooperação possibilita integrar competências e habilidades de várias organizações, culminando na obtenção dos recursos necessários para alcançar o sucesso no processo de internacionalização, especialmente das pequenas empresas (AMAL; FREITAG FILHO; MIRANDA, 2008; FENSTERSEIFER, 2000; FREEMAN; EDWARDS; SCHRODER, 2006, JOHNSEN; JOHNSEN, 1999; JOHANSON; VAHLNE, 1990).

Nesta perspectiva é possível afirmar que a internacionalização ocorre por meio de um processo interativo das vantagens competitivas da própria empresa a vantagens oriundas da rede na qual a organização está inserida
(ANDERSEN; BUVIK, 2002; JOHANSON; MATTSSON, 1988; SEPPO, 2007).

Neste sentido, Levy, Mota e Wermelinger (2009) destacam que as conexões interorganizacionais possibilitam obter diversos recursos facilitadores do processo de internacionalização, a saber: desenvolver e lançar novos produtos em menos tempo; produzir em escala; padronizar produtos e alcançar a qualidade necessária; adequar-se tecnologicamente; trocar informações e conhecimentos; fazer marketing conjunto; acessar informações do mercado local e gerar novas oportunidades de negócios.

A pesquisa de Alvarenga e Balestrin (2009) ratifica o posicionamento de que a cooperação contribui para a internacionalização, ao evidenciar em um estudo qualitativo no Vale da Eletrônica (Pólo de produtos eletroeletrônicos) localizado no Sul de Minas Gerais, que as pequenas e médias empresas, em parceria com Governo de Minas Gerais, a INATEL (Instituto Nacional de Telecomunicações), a SINDVEL (Sindicato das Indústrias de Aparelhos Elétricos, Eletroeletrônicos e Similares do Vale da Eletrônica) e o SEBRAE (Serviço Brasileiro de Apoio a Micro e Pequenas Empresas), alcançaram a participação em missões em outros países, ações coletivas para inovação tecnológica e capacitação técnico-acadêmica, resultando no crescimento do nível de internacionalização das PMEs a partir do suporte da rede.

Na mesma linha de análise, o estudo de Garcia, Lima e Carvalho (2010) envolvendo pequenas empresas do grupo Brazilian Health Products (BHP) do setor médico odontológico de Ribeirão Preto-SP, concluiu que através da parceria firmada entre estas organizações e a Agência Nacional de Promoção de Exportações e Investimentos (APEXBRASIL) foi possível desenvolver diversas ações, que proporcionaram a inserção destes empreendimentos no mercado externo, destacando a pesquisa de mercado, bem como a busca de outras informações gerais e comuns a todas as empresas (procedimentos para a certificação de produtos e atendimento de normas técnicas e regulamentos internacionais, adaptações necessárias aos produtos, adaptações na embalagem), registro de marca, promoção comercial e campanhas institucionais.

A pesquisa de Zen et. al. (2013), ao analisar o desenvolvimento de recursos no âmbito de uma rede interorganizacional do setor vinícola e a influência destes recursos no processo de internacionalização das empresas, evidenciou que a rede tem colaborado para a geração de recursos intangíveis, tais como a reputação do vinho brasileiro, o conhecimento do mercado e o fluxo de informação entre os participantes, contribuindo para a atuação internacional das empresas que fazem parte da rede.

Diante do exposto é possível afirmar que as pequenas e médias empresas podem superar as dificuldades da internacionalização, por meio de redes e cooperação, as quais proporcionam compartilhamento dos recursos (fi- 
nanceiro, tecnológico, humano, operacional) necessárias para as operações no mercado externo.

\section{Modelo Analítico}

Visando alcançar maior confiabilidade nesta pesquisa, a qual busca analisar como a rede de cooperação contribui para a internacionalização de pequenas e médias empresas, foi adotado o modelo analítico adaptado de Brito (1993), descrito na figura 1.

Neste modelo, Brito (1993) indica que a coordenação de atividades e conjugação de esforços coletivos, pode levar a obtenção de vantagens operacionais e estratégicas que impactam positivamente no processo de internacionalização da firma, conforme descrito abaixo.
Escala, gerada pelo de maior volume de produção e, consequentemente, reduções significativas nos custos unitários, bem como, através de compartilhamento de despesas na área comercial e maior força negocial em relação a fornecedores, clientes e instituições financeiras; (2) Sinergia, uma vez que a utilização de recursos complementares e anteriormente subaproveitados poderá dar origem a sinergias na área produtiva (partilha de equipamentos e instalações), na área comercial (partilha de redes de distribuição, forças de vendas e campanhas promocionais), na área técnica (partilha de know-how), dentre outras e (3) Economias de Experiência, pois à medida que uma unidade econômica ganha experiência na produção de um bem, o seu custo unitário tende a baixar em virtude da aprendizagem e de alguma inovação em nível de processos.

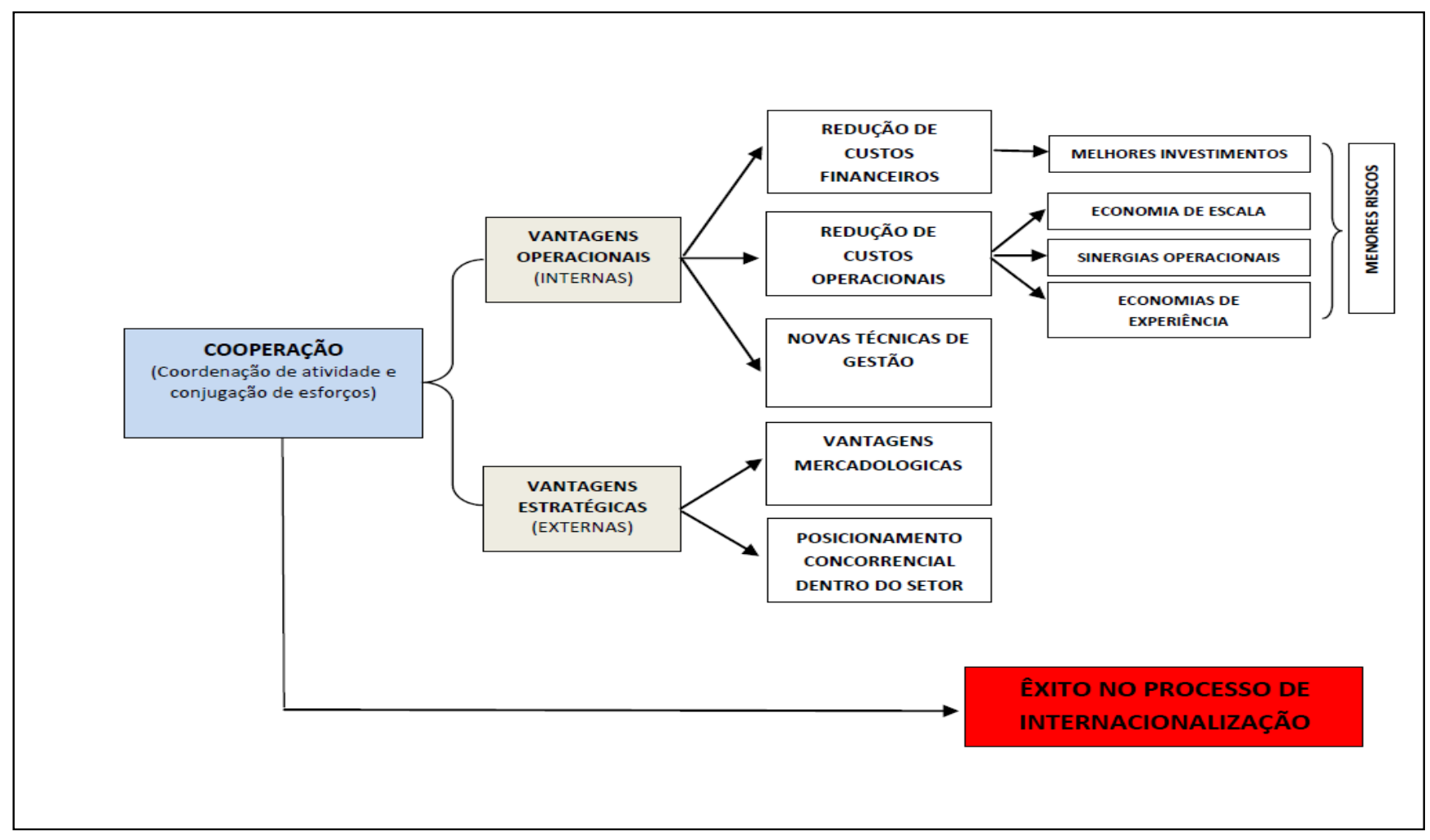

Figura 01 - Modelo de Pesquisa - Vantagens da Cooperação para a Internacionalização Fonte: Elaborado pelos autores (2013), com base em Brito (1993).

No âmbito das vantagens operacionais, a cooperação proporciona menores custos financeiros por meio do acesso a melhores investimentos, mediante a partilha das despesas (custo com equipamentos, formação de pessoal e prospecção e promoção no mercado) pelas várias entidades associadas, o que possibilita que cada uma delas invista menos em relação ao que seria investido caso viesse a lançar esse projeto de forma isolada.

A continuação, a cooperação também condiciona a redução de custos operacionais via: (1) Economia de
Brito (1993) esclarece em seguida que ao grupar a redução de custos financeiros e operacionais, observa-se que a cooperação possa ser uma opção menos arriscada do que investir isoladamente na internacionalização.

Neste contexto, o mesmo autor explica que as práticas coletivas interfirmas de produção e execução de tarefas que induzem a implantação de novos métodos de gestão na empresa associadas à rede e formas de organização adequadas à atuação no mercado externo.

No que concerne às vantagens estratégicas, verifica-se 
que a cooperação entre empresas direciona a obtenção de um conjunto de benefícios mercadológicos a exemplo da junção de firmas para reforço da quota de mercado; penetração em novos segmentos de mercado e/ou áreas geográficas específicas; aquisição de novas tecnologias que propiciem uma redução de custos, reforço da qualidade e/ou aumento da capacidade; produção e comercialização de novos produtos originados, nomeadamente, de um esforço conjunto de pesquisa e desenvolvimento.

Outro fator positivo da cooperação refere-se à possibilidade de melhor posicionamento frente à concorrência em virtude da obtenção de forças para atuar contra empresas de maior capacidade dentro de um determinado setor, ou do combate a forças que impactam as organizações, a exemplo do poder de barganha dos fornecedores.

Desta forma, a associação entre empresas permite reduzir custos e riscos, reforçar a imagem institucional e estabelecer-se de forma competitiva, ampliando as chances de êxito do processo de internacionalização.

A continuação apresenta os procedimentos metodológicos que nortearam a aplicação do modelo analítico descrito acima.

\section{Procedimentos Metodológicos}

Para atingir os objetivos traçados neste artigo, evidenciou-se como melhor estratégia de pesquisa, a adoção de um estudo qualitativo, a qual segundo Godoy (1995) e Richardson (1999) pauta-se na análise aprofundada de um fenômeno a partir da interpretação de determinadas informações, de modo a permitir a compreensão de processos dinâmicos, além de possibilitar elencar elementos ainda desconhecidos.

Para operacionalizar o tipo de pesquisa adotado, utilizou-se o método estudo de caso, por se mostrar como mais adequado, uma vez que Yin (2005) defende a adoção desta estratégia quando se deseja explorar uma ou poucas entidades (casos), por meio de coleta de informações dentro de um determinado contexto, buscando analisar uma situação de forma pormenorizada, ou seja, conforme proposto neste artigo.

O caso avaliado trata-se da Rede de Cooperação da Cadeia Produtiva de Petróleo e Gás em Sergipe - Rede Petrogas, o que caracteriza um estudo de caso único. É importante destacar que o caso escolhido foi considerado revelador para o escopo desta pesquisa, em virtude de satisfazer plenamente o entendimento da problematização traçada neste estudo.

Apesar do estudo ter sido de caso único, foram utilizadas múltiplas fontes de evidencias, a fim de coletar os dados necessários para alcançar os objetivos do artigo, conforme preconizam os autores Creswell (2002) e Saunders, Lewis e Thornill (2000).

Os dados que compõem o estudo do caso foram coletados por meio de entrevista semiestruturada, com a gestora da rede Petrogas-SE e o gestor de cada empresa internacionalizada associada à referida rede, no total de três organizações.

Destaca-se que a Rede Petrográs possui três empresas internacionalizadas, as quais foram alcançadas nesta pesquisa, e cujo perfil encontra-se descrito no quadro 03.

As entrevistas foram realizadas no mês de agosto do ano de 2013, como duração de aproximadamente 30 minutos. Os pesquisadores elaboraram dois roteiros de entrevista. O primeiro foi aplicado à gestora da Rede Petrogas-SE contendo indagações sobre a estruturação da rede e as suas principais ações para a internacionalização dos seus associados. O segundo questionário foi aplicado a cada um dos gestores das empresas internacionalizadas, buscando identificar o perfil da empresa e as contribuições da rede para a sua internacionalização.

Os roteiros de pesquisa foram estruturados com base nas categorias analíticas descritas no quadro 2 abaixo, elaboradas com base no modelo analítico adaptado de Brito (1993).

Além das entrevistas, adotou-se como fonte de evidências a análise de documentos. Os documentos analisados no estudo contemplaram: site da rede Petrogas, site das empresas internacionalizadas da rede Petrogás, portfólios (catálogos) das empresas avaliadas e documentos oficiais públicos (Relatórios da Balança Comercial Nacional e Local, Tabelas de Exportadores, Gráficos de Participação no Mercado Externo), disponibilizados pelo Ministério do Desenvolvimento, Indústria e Comércio Exterior - MDIC e Secretaria de Estado do Desenvolvimento Econômico e da Ciência e Tecnologia de Sergipe - SEDETEC.

Para analisar os dados coletados nas entrevistas foi adotado o método análise de conteúdo, o qual segundo Bardin (2008) corresponde à análise das comunicações, neste caso, os textos transcritos das entrevistas, com o propósito de inferir a partir de trechos, orações ou frases extraídas das respostas dos entrevistados, as informações necessárias para atender aos objetivos elencados no estudo.

Os dados coletados nas entrevistas e documentos analisados foram confrontados com o referencial teórico deste artigo, conforme exposto no próximo capitulo.

\section{Descrição e Análise do caso}

A seguir encontram-se a descrição e análise do caso à luz da fundamentação teórica acima apresentada, seguindo as categorias analíticas estabelecidas no artigo, as quais foram elaboradas com base no modelo analítico adaptado de Brito (1993).

\subsection{Perfil da rede Petrogas}

A Rede Petrogás no Estado de Sergipe foi criada em 2003, pelo SEBRAE/SE em parceria com a Petrobras, com o propósito de promover o desenvolvimento econômico do Estado através da integração de ações que visam 
Quadro 1 - Fonte de Evidências

\begin{tabular}{|c|c|l|}
\hline $\begin{array}{c}\text { TÉCNICA DE } \\
\text { COLETA DE DADOS }\end{array}$ & $\begin{array}{c}\text { FONTE DE } \\
\text { EVIDÊNCIA }\end{array}$ & \multicolumn{1}{c|}{ OBJETIVO } \\
\hline Entrevista & $\begin{array}{c}\text { Gestora da Rede } \\
\text { Petrogás }\end{array}$ & $\begin{array}{l}\text { Traçar o perfil da rede } \\
\text { Identificar os benefícios e vantagens da } \\
\text { rede para a internacionalização das PMES }\end{array}$ \\
\hline Entrevista & $\begin{array}{c}\text { Gestores das PMEs } \\
\text { internacionalizadas }\end{array}$ & $\begin{array}{l}\text { Traçar o perfil da empresa } \\
\text { Identificar os benefícios e vantagens da } \\
\text { rede para a internacionalização das PMES, } \\
\text { na visão do associado à rede. }\end{array}$ \\
\hline Documentos & Sites da rede \\
Sites das empresas & $\begin{array}{l}\text { Ratificar o perfil da rede e das empresas } \\
\text { Validar as informações técnicas e oficiais } \\
\text { disponibilizadas pelos empresários; } \\
\text { Confirmar os benefícios e vantagens } \\
\text { Pisponibilizados pela rede Petrogras para } \\
\text { a internacionalização das PMEs. }\end{array}$ \\
\hline
\end{tabular}

Fonte: Elaborado pelos autores (2013)

Quadro 2 - Questões de Pesquisa, Categorias Analíticas e Elementos de Análise

\begin{tabular}{|c|c|c|}
\hline QUESTÕES DE PESQUISA & $\begin{array}{l}\text { CATEGORIAS } \\
\text { ANALITICAS }\end{array}$ & ELEMENTOS DE ANÁLISE \\
\hline Qual o perfil da rede Petrógas? & Perfil da rede Petrogas & $\begin{array}{l}\text { - Objetivos } \\
\text { - Associados } \\
\text { - Ações }\end{array}$ \\
\hline $\begin{array}{l}\text { Qual o perfil das empresas } \\
\text { internacionalizadas da rede } \\
\text { Petrógas? }\end{array}$ & $\begin{array}{c}\text { Perfil das empresas } \\
\text { internacionalizadas da Rede } \\
\text { Petrogas }\end{array}$ & $\begin{array}{l}\text { - Campo de atuação } \\
\text { - Número de empregados } \\
\text { - Fundação } \\
\text { - Serviços/produtos comercializados } \\
\text { no mercado internacional } \\
\text { - Identificação da rede associada }\end{array}$ \\
\hline $\begin{array}{l}\text { Como a rede Petrogás } \\
\text { contribuiu para o processo de } \\
\text { internacionalização das } \\
\text { empresas associadas à rede? }\end{array}$ & $\begin{array}{c}\text { Contribuição da rede Petrogas } \\
\text { para a internacionalização }\end{array}$ & $\begin{array}{l}\text { - Compartilhamento de riscos } \\
\text { - Compartilhamento de recursos } \\
\text { - Pesquisas, certificações e } \\
\text { treinamentos. }\end{array}$ \\
\hline
\end{tabular}

Fonte: Elaborado pelos autores (2013)

inserir as pequenas e médias empresas sergipanas no comércio local, nacional e internacional da cadeia produtiva de petróleo e gás.

Esta rede faz parte do "Programa Cadeia Produtiva do Petróleo e Gás", o qual se encontra atuando em rede e provendo ações correlatas em onze Estados (Rio de Janeiro, AM, Ceará, Rio Grande do Norte, Sergipe,
Alagoas, Bahia, Espirito Santo, Minas Gerais, Paraná e Rio Grande do Sul). Atualmente, a nível nacional, mais de 600 empresas estão participando do programa.

De acordo com Araújo, Pellegrin e Fernandes (2005) pode-se justificar a criação destas redes em virtude recente abertura do setor de petróleo, tanto no Brasil, quanto em outros países, o que aumentou a demanda 
por bens e serviço ao longo da cadeia petrolífera nacional e internacional.

No Estado de Sergipe, rede Petrogas envolve oito municípios sergipanos, a saber: Aracaju (cidade polo), Itaporanga D'Ajuda, Riachuelo, São Cristovão, Santo Amaro das Brotas, Estância, Carmópolis e Japaratuba e possui 184 empresas associadas de diversos segmentos empresariais. Deste universo, três PMEs encontram-se atuando no mercado externo.

O próximo capítulo apresenta o perfil das empresas internacionalizadas da rede.

\subsection{Perfil das empresas internacionalizadas da Rede Petrogas}

Hoje, a rede Petrogras possui três empresas atuando no mercado internacional, cujos dados encontra-se descrito no quadro 3 abaixo.
A pesquisa revelou que as empresas A e B atuam na área de prestação de serviços para a cadeia petrolífera. Destaca-se ainda que a empresa " $B$ " foi à primeira firma da América Latina a ser certificada para a prestação de serviço de capacitação na área de controle de poço nas atividades de perfuração, nos níveis introdutório, fundamental e supervisão.

A empresa " $C$ ", por sua vez, atua na área de fabricação de produtos químicos destinados a indústrias do mesmo ramo. Esta empresa possui sede em Aracaju/ SE e conta com duas filiais, uma no estado da Paraíba e outra no estado da Bahia.

Destaca-se que as três empresas ingressaram no mercado externo a partir de 2004 após a criação da rede Petrogas.

Em seguida, observa-se que os gestores das empresas avaliadas confirmaram que o suporte da rede foi indispensável para ingressar no mercado externo, corroborando o

Quadro 3 - Perfil das empresas internacionalizadas da rede Petrogas-SE

\begin{tabular}{|c|c|c|c|}
\hline CARACTERÍSTICAS & EMPRESA A & EMPRESA B & EMPRESA C \\
\hline Fundação & 2000 & 1998 & 1993 \\
\hline Localização (Sede) & Aracaju/SE & Aracaju/SE & Aracaju/SE \\
\hline $\begin{array}{ll}\text { Quantidade } & \text { de } \\
\text { Empregados } & \end{array}$ & 90 & 20 & 120 \\
\hline Porte Empresarial & Médio porte & Pequeno Porte & Médio porte \\
\hline $\begin{array}{l}\text { Atividade } \\
\text { Empresarial }\end{array}$ & $\begin{array}{l}\text { Prestação de Serviço de } \\
\text { Consultoria na área de } \\
\text { Petróleo, Elaboração de } \\
\text { Projeto e Fiscalização de } \\
\text { Operação de Perfuração } \\
\text { com Sonda e o } \\
\text { Desenvolvimento de } \\
\text { equipamentos na área } \\
\text { de perfuração. }\end{array}$ & $\begin{array}{l}\text { Prestação de serviço de } \\
\text { treinamento e } \\
\text { consultoria na área de } \\
\text { exploração de petróleo e } \\
\text { desenvolvimento de } \\
\text { software simulador de } \\
\text { segurança e controle de } \\
\text { exploração de poços em } \\
\text { águas profundas. }\end{array}$ & $\begin{array}{l}\text { Fabricação de } \\
\text { químicos da área de } \\
\text { petróleo } \\
\text { espumantes; } \\
\text { antiespumantes; } \\
\text { bactericidas e outros). }\end{array}$ \\
\hline $\begin{array}{l}\text { Estratégia de } \\
\text { Internacionalização }\end{array}$ & $\begin{array}{l}\text { Exportação direta dos } \\
\text { serviços }\end{array}$ & $\begin{array}{l}\text { Exportação direta das } \\
\text { licenças dos softwares. }\end{array}$ & $\begin{array}{l}\text { Exportação direta dos } \\
\text { produtos fabricados. }\end{array}$ \\
\hline Países atendidos & $\begin{array}{l}\text { Colômbia, } \\
\text { Venezuela, } \\
\text { Argentina } \\
\text { Arábia Saudita }\end{array}$ & $\begin{array}{l}\text { Argentina, } \\
\text { Bolívia, } \\
\text { Estados Unidos } \\
\text { Inglaterra }\end{array}$ & $\begin{array}{l}\text { Argentina, } \\
\text { Chile, } \\
\text { Estados Unidos, } \\
\text { Colômbia, } \\
\text { Venezuela } \\
\text { Arábia Saudita }\end{array}$ \\
\hline $\begin{array}{l}\text { Motivos para a } \\
\text { Internacionalização }\end{array}$ & $\begin{array}{l}\text { Busca de novos } \\
\text { mercados; } \\
\text { Procura de maior } \\
\text { visibilidade no mercado } \\
\text { local. } \\
\end{array}$ & $\begin{array}{l}\text { Aumentar a quantidade } \\
\text { de clientes. }\end{array}$ & $\begin{array}{l}\text { Busca de novos } \\
\text { mercados; } \\
\text { Ampliação } \\
\text { quantitativo de clientes. }\end{array}$ \\
\hline
\end{tabular}

Fonte: Dados da pesquisa (2013). 
entendimento dos autores Chetty e Angdal (2007), Chetty e Campbell-Hunt (2004) e Johnsen e Johnsen (1999) de que a integração empresarial auxilia a superar os obstáculos que dificultam a internacionalização das PMEs.

O próximo capitulo expõe detalhadamente as contribuições da rede para a internacionalização das pequenas e médias empresas.

\subsection{Contribuições da Rede Petrogas-SE para o Processo de Internacionalização}

De acordo com a gestora da rede Petrogas, para subsidiar a internacionalização das PMEs, a citada rede firmou parcerias com a Federação das Indústrias de Sergipe - FIES e com o Serviço Nacional de Aprendizagem Industrial - SENAI e o Instituto Euvaldo Lodi- IEL, os quais promoveram palestras, cursos, treinamento e capacitações sobre como atuar no mercado externo. A entrevistada esclareceu que por meio desta ação, as empresas hoje internacionalizadas, conseguiram obter os conhecimentos necessários para ingressar na atividade internacional.

Outra parceria de destaque da rede é o Serviço de Apoio às Micro e Pequenas Empresas de Sergipe - SEBRAE/SE, que também influenciou positivamente neste processo, mediante consultorias e estudos especializados em internacionalização de micro e pequenas empresas, os quais possibilitaram às firmas escolher o mercado de atuação no exterior.

Ainda neste quesito, outro parceiro de importante foi o Sergipe Parque Tecnológico - SERGIPETEC, com apoio em pesquisa e desenvolvimento de produtos da cadeia de petróleo e gás.

E por fim, salienta-se a parceria do Banco do Nordeste do Brasil - BNB e do Banco do Brasil com linhas de créditos específicas para os pequenos empreendimentos da rede Petrogás que optam pela internacionalização.

$\mathrm{O}$ fato da rede Petrogas contar com os parceiros acima citados, confirma o posicionamento dos autores Balestrin, Verschoore e Reyes Junior (2010) e Castro, Bulgacov e Hoffmann (2011) de que as redes cooperativas são acordos feitos entre atores econômicos (empresas) e que podem envolver atores não econômicos (apoio governamental) que interagem com o objetivo de alcançar resultados sinérgicos.

Além do apoio dos vários órgãos acima citados, os gestores das empresas internacionalizadas destacaram que a rede Petrogas/SE também contribuiu diretamente para o processo de internacionalização subsidiando a obtenção de certificações, consideradas essenciais para atuar no mercado internacional. Veja os relatos:

[...] através da rede foi possível ratear com outras empresas os custos com as certificações ISO 9001 - Qualidade; ISO 14001 - Meio ambiente; OHSAS 18001 - Saúde e Segurança Ocupacional [...] Sem a rede, ou seja, sozinhos, provavelmente não seria possível obter estas certificações em virtude do alto custo e dos investimentos necessários para consegui-las (Empresário "A"). A rede Petrogas facilitou o processo de internacionalização através de várias ações, em especial: o suporte para obtenção das certificações de qualidade e segurança, indispensável para o mercado externo (Empresário " $\mathrm{C}$ ").

Outra contribuição da rede destacada pelos empresários entrevistados foi à viabilização da participação em feiras internacionais como Brazil of Shore, Rio Oil \& Gas e Vitoria Oil \& Gas, as quais contribuíram para a divulgação das empresas, acesso a novos clientes internacionais, troca de informações e conhecimentos, além do e fortalecimento da imagem da empresa. Veja os depoimentos abaixo:

[...] a rede Petro (Nacional) é parceira dos eventos Brazil of Shore, Rio Oil \& Gas e Vitoria Oil e Gás. A rede Petrogas (Sergipe) facilitou a nossa participação nestes eventos (Empresário A). [...] estas feiras contam com a participação de empresas de diversos países e nestes eventos tivemos a oportunidade de divulgar o nosso trabalho, fazer contato com potenciais clientes e trocar informações e experiência com empresas tanto nacionais como internacionais (Empresário B). [...] a partir das feiras foi possível demonstrar em nosso país os nossos produtos e serviços para os clientes de fora, evitando com isso os custos com demonstração em loco, ou seja, no país do importador [...] de um modo geral, ser integrante da rede e participar dos eventos pela rede melhora a imagem da empresa para o futuro cliente e aumenta a nossa credibilidade (Empresário C).

Ao analisar os recursos disponibilizados pela rede Petrogas para a internacionalização das pequenas empresas, a luz do modelo analítico adaptado de Brito (1993), é possível observar que a referida rede proporcionou a obtenção de vantagens operacionais, por meio de menores custos financeiros ao disponibilizar a (1) partilha de despesas para a obtenção de certificação e (2) o acesso a melhores linhas de investimentos disponibilizadas pelos bancos parceiros da rede, configurando uma opção menos arriscada do que investir na internacionalização isoladamente.

No que concerne às vantagens estratégicas, verifica-se que a rede Petrogas proporcionou o acesso a benefícios mercadológicos via (1) consultorias e apoio para ingresso em novos mercados; (2) capacitação para atuar no mercado externo, (3) participação em feiras, as quais possibilitaram prospectar as firmas no mercado internacional (4) parceiras com órgãos especializados em pesquisas voltadas ao desenvolvimento de produtos visando atender o mercado externo.

A seguir, o quadro 4 apresenta a síntese das contribuições da rede Petrogas para a internacionalização das pequenas e médias empresas, com base modelo analítico adaptado de Brito (1993).

Face ao quadro acima, visualiza-se que as vantagens 
Quadro 4 - Contribuições da rede para a internacionalização

\begin{tabular}{|c|c|c|c|c|}
\hline & ATOR & CONTRIBUIÇÕES & VANTAGENS & TIPO \\
\hline & & $\begin{array}{l}\text { Partilha de despesas com } \\
\text { certificações }\end{array}$ & Operacional & $\begin{array}{l}\text { Menor Custo } \\
\text { Financeiro }\end{array}$ \\
\hline & Rede Petrogás & Participação em Feiras & Estratégica & Mercadológica \\
\hline & FIES; SENAI; IEL. & $\begin{array}{l}\text { Capacitação para atuar no } \\
\text { mercado externo }\end{array}$ & Estratégica & Concorrencial \\
\hline 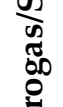 & SEBRAE/SE & $\begin{array}{l}\text { Consultorias e apoio para } \\
\text { ingresso em novos mercados }\end{array}$ & Estratégica & Mercadológica \\
\hline 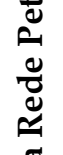 & SERGIPETEC & $\begin{array}{l}\text { Pesquisas para } \\
\text { desenvolvimento de } \\
\text { produtos }\end{array}$ & Estratégica & Mercadológica \\
\hline 常 & $\begin{array}{ll}\text { BNB - Banco do } \\
\text { Nordeste } \\
\text { Brasil }\end{array}$ & $\begin{array}{l}\text { Financiamentos e Linhas } \\
\text { Especiais de Crédito }\end{array}$ & Operacional & $\begin{array}{l}\text { Menor Custo } \\
\text { Financeiro }\end{array}$ \\
\hline & Banco do Brasil & $\begin{array}{l}\text { Financiamentos e Linhas } \\
\text { Especiais de Crédito }\end{array}$ & Operacional & $\begin{array}{l}\text { Menor Custo } \\
\text { Financeiro }\end{array}$ \\
\hline
\end{tabular}

Fonte: Dados da pesquisa (2013).

e benefícios oriundos da rede Petrogras induziram ao êxito do processo de internacionalização das PMEs associadas à rede, confirmando os argumentos de Amal, Freitag Filho e Miranda (2008), Balestrin e Vargas (2004), Dalmoro (2009), Fensterseifer (2000) e Freeman; Edwards e Schroder (2006) de que as limitações para o processo de internacionalização das PMEs podem ser superadas por meia da cooperação interorganizacional.

\section{Conclusões}

O presente artigo buscou por meio do método estudo de caso analisar como a Rede de Cooperação da Cadeia Produtiva de Petróleo e Gás em Sergipe contribui para a internacionalização das pequenas e médias empresas associadas à referida rede.

Em síntese, a pesquisa revelou que a rede Petrogas disponibilizou o acesso a diversos serviços especializados, tais como consultorias, treinamentos, pesquisa e desenvolvimento de produtos, por meio de parcerias firmadas entre órgãos governamentais.

Além destes serviços, as ações da rede Petrogas-SE conduziram redução de custos via compartilhamento de despesas entre associados; bem como o acesso a eventos de cunho estratégico para o setor petrolífero.

$\mathrm{O}$ estudo mostrou ainda que as PMEs só ingressaram na atividade internacional após a criação da citada rede. Este fato direciona ao entendimento de que sem os benefícios e vantagens advindos da Rede Petrogas, provavelmente estas empresas não teriam conseguido ingressar o mercado externo.

Em face destes resultados, a pesquisa apresenta como contribuições acadêmicas, a ratificação do pressuposto de que por meio da rede de cooperação interorganizacional as pequenas e médias empresas podem superar os fatores que impedem a sua

\section{Referências}

ALVARENGA, S. B.; BALESTRIN, A. Estratégias coletivas para internacionalização de pequenas e médias empresas. In.: ENCONTRO

DA ASSOCIAÇÃO NACIONAL DE PÓS-GRADUAÇÃO E PESQUISA EM ADMINISTRAÇÃO, 33., 2009, São Paulo. Anais... Rio de Janeiro: ANPAD, 2009.

AMAL, M.; FREITAG FILHO, A. R.; MIRANDA, C; M. S. Algumas evidências sobre o papel das redes de relacionamento e empreendedorismo na internacionalização das pequenas e médias empresas. Revista Faces de Administração, Belo Horizonte, v. 7, n. 1, p. 63-80, janeiro/março, 2008. 
AMATO NETO, J. Redes de cooperação produtiva e clusters regionais: oportunidades para as pequenas e médias empresas. São Paulo: Atlas, 2000.

ANDERSEN, O.; BUVIK, A. Firms' internationalization and alternative approaches to the international customer/market selection. International Business Review, Oxford, v.11, n.3, p.347-363, june, 2002.

ARAÚJO, Renato S. B. d; PELLEGRIN, Ivan De; FERNANDES, Elton. Articulação estratégica no setor petróleo e gás no Brasil - as redes de empresas e o fornecimento competitivo de bens e serviços. XXV Encontro Nac. de Eng. de Produção Porto Alegre, RS, Brasil, 29 out a 01 de nov de 2005

ARAGÃO, L. A; LOPES, C. S., ALVES JUNIOR, M. D. Redes de Cooperação de Pequenas e Médias Empresas: Os Benefícios Estratégicos em Uma Rede de Supermercados.In: ENCONTRO DE ESTUDOS ORGANIZACIONAIS DA ANPAD, 6., Florianópolis, 2010

BALESTRIN, A.; VARGAS, L. M. A dimensão estratégica das redes horizontais de PMEs: teorizações e evidências. RAC - Revista de Administração Contemporânea, Curitiba, v. 8, Ed. Espec., p. 203-228, 2004.

BALESTRIN, A.; VERSCHOORE, J. R.; REYESJUNIOR, E. O Campo de estudos sobre redes de cooperação interorganizacional no Brasil. RAC - Revista de Administração Contemporânea, Curitiba, v. 14, n.4, p.458-477, maio/junho, 2010.

BARDIN, L. Análise de conteúdo. Lisboa: Edições 70, 2008.

BEAMISH, P. W. The internationalization process for smaller Ontario firms: a research agenda. In: RUGMAN, A. Research in Global Business Management. Greenwich: JAI Press, 1990.

BOEHE, D. M., TONI, D. T. Modelo para internacionalização de empresas baseada em redes. In: SIMPÓSIO DE GESTÃO DA INOVAÇÃO TECNOLÓGICA. 24., 2006, Gramado, Anais... Gramado: ANPAD, 2006.

BRITO, C. M. Estratégias de Internacionalização e cooperação. Working paper nº 38, Faculdade de Economia do Porto, 1993.

CALDAS, P. T.; VASCONCELOS, A. C. F.; ANDRADE, E. O.; CÂNDIDO, G. A. Estratégias para internacionalização de empresas com atuação em redes: um estudo exploratório em um consórcio de exportação no setor de confecções. Revista GEPROS - Gestão da Produção, Operações e Sistemas, Bauru, v. 5, n. 3, p. 93-111, julho/ setembro, 2010.

CÂNDIDO, G. A. A formação de redes interorganizacionais como mecanismo para geração de vantagem competitiva e para promoção do desenvolvimento regional: o papel do estado e das políticas públicas neste cenário. REAd - Revista Eletrônica de Administração, Porto Alegre, vol. 8 n. 4, julho/agosto. 2002.

CASTRO, M.; BULGACOV, S.; HOFFMANN, V.E. Relacionamentos interorganizacionais e resultados: estudo em uma rede de cooperação horizontal da região central do Paraná. RAC - Revista de Administração Contemporânea, Curitiba, v. 15, n. 1, art. 2, p. 25-46, janeiro/fevereiro, 2011.

CHETTY, S.; AGNDAL, H. Social capital and its influence on changes in internationalization mode among small and medium-sized enterprises. Journal of International Marketing, Chicago, v. 15, n. 1, p. 1-29, 2007.

CHETTY, S.; CAMPBELL-HUNT, C. A strategic approach to internalization: a traditional versus a "born global" approach. Journal of International Marketing, Chicago, v. 12, n. 1, p. 57-81, 2004.

CORTEZIA, S. L. D.; SOUZA, Y. S. Uma análise sobre a internacionalização de pequenas empresas brasileiras da indústria de software. Brasilian Business Review. Vitória, v. 8, n. 4, p. 24-45, Out. Dez. 2011.

CRESWELL, J. Research design: qualitative e quantitative, and mixed approaches. 2nd ed. Thousand Oaks: Sage, 2002.

DALMORO, M. Internacionalização de empresas em redes horizontais: uma análise a partir do Projeto Setorial Integrado Wines from Brazil. 2009. 187 f. Dissertação (Mestrado em Administração) Programa de Pós-Graduação em Administração, Universidade Federal de Santa Maria, Santa Maria, 2009.

DALMORO, M.; WITTMANN, M. Processos de internacionalização em rede: cooperando para conquistar o mercado externo. Revista de Administração e Contabilidade da Unisinos, Porto Alegre, v. 8, n.3, p. 231-242, julho/setembro, 2011.

FENSTERSEIFER, J. E. Internacionalização e 
cooperação: dois Imperativos para a empresa do terceiro milênio. REAd - Revista Eletrônica de Administração, Porto Alegre, v. 6, n. 3, p. 1 - 9, outubro, 2000.

FLORIANI, D. E; FLEURY, M. T. O efeito do grau de internacionalização nas competências internacionais e no desempenho financeiro da PME brasileira. RAC - Revista de Administração Contemporânea, Curitiba, v. 16 n. 3, pp. 438 - 458, maio/junho, 2012.

FREEMAN, S.; EDWARDS, R.; SCHRODER, B. How Smaller Born-Global Firms Use Networks and Alliances to Overcome Constraints to Rapid Internationalization. Journal of International Marketing, Chicago, v. 14, n.3, p.33-63, 2006.

GARCIA, S. F. A; LIMA, G. B.; CARVALHO, D. T. Redes interorganizacionais de cooperação para a internacionalização. REGE - Revista de Gestão, São Paulo, v.17, n.2, p. 209-224, abril/junho, 2010.

GODOY, A. S. Pesquisa qualitativa: tipos fundamentais. RAE - Revista de Administração de Empresas, São Paulo, v. 35, n.3, p. 20-29, maio/ Junho, 1995.

HOLLENSTEIN, H. Determinants of international activities: are SMEs different? Small Business Economics, v. 24, n. 5, p. 431-450, june, 2005.

JOHANSON, J.; MATTSON, L. Internationalization in industrial systems: a networkapproach. In. HOOD, N. e VAHLNE, J. (eds.). Strategies in global competition. New York: Croom Helm, 1988.

JOHANSON, J.; VAHLNE, J.E. The mechanism of Internationalization. International Marketing Review, v. 7, n. 4, p.11-24, 1990

JOHNSEN, R. E.; JOHNSEN, T. E. International market development through networks. the case of the Ayrshire knitwear sector. International Journal of Entrepreneurial Behavior \& Research. v. 5, n. 6, p. 297-312, 1999.

JUCHNIEVSK, L. C,; SOARES. I. T. Consórcio de exportação como alternativa de internacionalização para pequenas empresas Revista de Administração, v. 11, n. 20, p. 70-84, Dez. 2013

LEVY, B. P.; MOTA, M. C.; WERMELINGER, M. B. O uso de networks no processo de internacionalização: aplicação a pequenas e médias empresas. In: ENCONTRO DA ASSOCIAÇÃO NACIONAL DE PÓS-GRADUAÇÃO E PESQUISA
EM ADMINISTRAÇÃO, 33., 2009, São Paulo. Anais... São Paulo: ANPAD, 2009.

MACCHIA, E. L.; WASSERMAN, J. C.; BINSZTOK, J;. LIMA, G. B. A. As estratégias de internacionalização da indústria do petróleo. ENGEVISTA, v. 16, n. 2, p.266-281, Junho 2014.

MARTINS, F. A; SANTANA, J. R. Estratégia de atuação em uma rede de micro, pequenas e médias empresas (MPMES) no setor de petróleo e gás (P\&G): uma análise dos resultados no estado de Sergipe. In.: ENCONTRO DE ESTUDOS EM EMPREENDEDORISMO E GESTÃO DE PEQUENAS EMPRESAS - EGEPE, 8., 2014, Goiânia. Anais... São Paulo: ANEGEPE, 2014.

MINISTÉRIO DO DESENVOLVIMENTO, INDÚSTRIA E COMÉRCIO EXTERIOR - MDIC. Comércio Exterior. In: Estatística comercial Brasileira SECEX/DEPLA. 2012. Disponível em: <http://www.desenvolvimento.gov.br/sitio/interna/ index.php?area $=5>$. Acesso em 30 de janeiro de 2013.

NEUMAN, Lawrence W. Social research methods: qualitative and quantitative approaches. 3. ed. Boston: Allyn \& Bacon, Cap 2 -Dimension of Research. p 18 a 35, 1997

OLIVEIRA, M. F; MARTINELLI, D. P. A internacionalização de pequenas empresas através de consórcios de exportação inseridos em clusters industriais: uma relação recíproca de contribuição. Revista Internacional de Desenvolvimento Local, Campo Grande, v. 6, n.10, p.55-63, março, 2005.

PIRES, E.R.O; MACHADO NETO, A.J. Redes de cooperação como alternativa para o desenvolvimento local: a indústria calçadista Francana. Revista Eletrônica de Administração (Online), v. 11, n.2, edição 21, julho/dezembro, 2012.

REIS, J.A,; EL-KOUBA, A.;QUANDT, C.O.; CRUZ, J.A.W.; MARTINS, T.S. Cooperação e desenvolvimento: estudo de caso em uma rede de cooperação. Revista Gerenciais, São Paulo, v.7, n.1, p.21-28, junho, 2008.

RICHARDSON, R. J. Pesquisa social: métodos e técnicas. 3. ed. São Paulo: Atlas, 1999.

SAUNDERS, M; LEWIS, P.; THORNILL, A. Research Methods for Business Students. 2.ed. Harlow, England: Pearson Education, 2000. Cap 4- Pg 84 a 95 
SEPPO, M. The role of business networks in the internationalization of Estonian Chemical Industry Enterprises. University of Tartu, n.7, 2007.

VERDU, F. C. A. Internacionalização de uma pequena empresa. In: ENCONTRO NACIONAL DOS PROGRAMAS DE PÓSGRADUAÇÃO EM ADMINISTRAÇÃO, 35., 2011, Rio de Janeiro, Anais... Rio de Janeiro: ANPAD, 2011.

VERSCHOORE, J. R.; BALESTRIN, A. Fatores relevantes para o estabelecimento de redes de cooperação entre empresas do Rio Grande do Sul. RAC - Revista de Administração Contemporânea, Curitiba, v. 12, p. 1043-1069, outubro/dezembro, 2008.

VINHAS, A. P. L. C.; BECKER, G. V. Redes de cooperação interorganizacional: $\mathrm{O}$ caso da rede macsul. Revista Análise, Porto Alegre, v. 17, n. 1, p. 167-184, janeiro/julho, 2006.

YIN, R. Estudo de caso: planejamento e métodos. 3. ed. Porto Alegre: Bookman, 2005.

ZAHRA, S. A., UCBASARAN, D., NEWEY, L. R. Social knowledge and SMEs' innovative gains from internationalization. European Management Review, v. 6, n. 2, p. 81-93, summer, 2009.

ZEN, A.C.; DALMORO; M.; FENSTERSEIFER, J. E.; WEGNER, D. O desenvolvimento de recursos em redes interorganizacionais e o processo de internacionalização: o caso Wines of Brasil. RIAE Revista Ibero-Americana de Estratégia , São Paulo, v. 12, n. 1, p. 107-130, jan./mar. 2013 